\title{
GRAPH METRIC LEARNING VIA GERSHGORIN DISC ALIGNMENT
}

\author{
Cheng Yang ${ }^{\dagger}$, Gene Cheung ${ }^{\dagger}$, Wei Hu$u^{\ddagger}$ \\ ${ }^{\dagger}$ Department of Electrical Engineering \& Computer Science, York University, Toronto, Canada \\ ${ }^{\ddagger}$ Wangxuan Institute of Computer Technology, Peking University, Beijing, China
}

\begin{abstract}
We propose a general projection-free metric learning framework, where the minimization objective $\min _{\mathbf{M} \in \mathcal{S}} Q(\mathbf{M})$ is a convex differentiable function of the metric matrix $\mathbf{M}$, and $\mathbf{M}$ resides in the set $\mathcal{S}$ of generalized graph Laplacian matrices for connected graphs with positive edge weights and node degrees. Unlike low-rank metric matrices common in the literature, $\mathcal{S}$ includes the important positivediagonal-only matrices as a special case in the limit. The key idea for fast optimization is to rewrite the positive definite cone constraint in $\mathcal{S}$ as signal-adaptive linear constraints via Gershgorin disc alignment, so that the alternating optimization of the diagonal and offdiagonal terms in $\mathbf{M}$ can be solved efficiently as linear programs via Frank-Wolfe iterations. We prove that left-ends of the Gershgorin discs can be aligned perfectly using the first eigenvector $\mathbf{v}$ of $\mathbf{M}$, which we update iteratively using Locally Optimal Block Preconditioned Conjugate Gradient (LOBPCG) with warm start as diagonal / off-diagonal terms are optimized. Experiments show that our efficiently computed graph metric matrices outperform metrics learned using competing methods in terms of classification tasks.
\end{abstract}

Index Terms - Metric Learning, graph signal processing

\section{INTRODUCTION}

Given a feature vector $\mathbf{f}_{i} \in \mathbb{R}^{K}$ per sample $i$, a metric matrix $\mathbf{M} \in$ $\mathbb{R}^{K \times K}$ defines the feature distance (Mahalanobis distance [1]) between two samples $i$ and $j$ in a feature space as $\left(\mathbf{f}_{i}-\mathbf{f}_{j}\right)^{\top} \mathbf{M}\left(\mathbf{f}_{i}-\mathbf{f}_{j}\right)$, where $\mathbf{M}$ is commonly assumed to be positive definite (PD). Metric learning - identifying the best metric $\mathbf{M}$ minimizing a chosen objective function $Q(\mathbf{M})$ subject to $\mathbf{M} \succ 0$-has been the focus of many recent machine learning research efforts $[2,3,4,5,6]$.

One key challenge in metric learning is to satisfy the positive (semi-)definite (PSD) cone constraint $\mathbf{M} \succ 0$ ( $\mathbf{M} \succeq 0$ ) when minimizing $Q(\mathbf{M})$ in a computation-efficient manner. A standard approach is alternating gradient-descent / projection (e.g., proximal gradient (PG) [7]), where a descent step $\boldsymbol{\alpha}$ from current solution $\mathbf{M}^{t}$ at iteration $t$ in the direction of the negative gradient $-\nabla Q\left(\mathbf{M}^{t}\right)$ is followed by a projection $\operatorname{Pr}()$ back to the PSD cone, i.e., $\mathbf{M}^{t+1}:=\operatorname{Pr}\left(\mathbf{M}^{t}-\boldsymbol{\alpha} \nabla Q\left(\mathbf{M}^{t}\right)\right)$. However, projection $\operatorname{Pr}()$ typically requires eigen-decomposition of $\mathbf{M}$ and soft-thresholding of its eigenvalues, which is computation-expensive.

Recent methods consider alternative search spaces of matrices such as sparse or low-rank matrices to ease optimization [3, 4, 5, 8, 9]. While efficient, the assumed restricted search spaces

Gene Cheung acknowledges the support of the NSERC grants [RGPIN2019-06271], [RGPAS-2019-00110].

Wei Hu acknowledges the support of National Natural Science Foundation of China (61972009) and Beijing Natural Science Foundation (4194080) often degrade the quality of sought metric $\mathbf{M}$ in defining the Mahalanobis distance. For example, low-rank methods explicitly assume reducibility of the $K$ available features to a lower dimension, and hence exclude the simple yet important weighted feature metric case where $\mathbf{M}$ contains only positive diagonal entries [10], i.e., $\left(\mathbf{f}_{i}-\mathbf{f}_{j}\right)^{\top} \mathbf{M}\left(\mathbf{f}_{i}-\mathbf{f}_{j}\right)=\sum_{k} m_{k, k}\left(f_{i}^{k}-f_{j}^{k}\right)^{2}, m_{k, k}>0, \forall k$. We show in our experiments that computed metrics by these methods may result in inferior performance for selected applications.

In this paper, we propose a metric learning framework that is both general and projection-free, capable of optimizing any convex differentiable objective $Q(\mathbf{M})$. Compared to low-rank methods, our framework is more encompassing and includes positive-diagonal metric matrices as a special case in the limit. The main idea is as follows. First, we define a search space $\mathcal{S}$ of general graph Laplacian matrices [11], each corresponding to a connected graph with positive edge weights and node degrees. The underlying graph edge weights capture pairwise correlations among the $K$ features, and the self-loops designate relative importance among the features.

Assuming $\mathbf{M} \in \mathcal{S}$, we next rewrite the PD cone constraint as signal-adaptive linear constraints via Gershgorin disc alignment [12, 13]: i) compute scalars $s_{k}$ 's from previous solution $\mathbf{M}^{t}$ that align the Gershgorin disc left-ends of matrix $\mathbf{S M}^{t} \mathbf{S}^{-1}$, where $\mathbf{S}=$ $\operatorname{diag}\left(s_{1}, \ldots, s_{K}\right)$, ii) derive scaled linear constraints using $s_{k}$ 's to ensure PDness of the next computed metric $\mathbf{M}^{t+1}$ via the Gershgorin Circle Theorem (GCT) [14]. Linear constraints mean that our proposed alternating optimization of the diagonal and off-diagonal terms in $\mathbf{M}$ can be solved speedily as linear programs (LP) [15] via Frank-Wolfe iterations [16]. We prove that for any metric $\mathbf{M}^{t}$ in $\mathcal{S}$, using scalars $s_{k}=1 / v_{k}$ can perfectly align Gershgorin disc leftends for matrix $\mathbf{S M}^{t} \mathbf{S}^{-1}$ at the smallest eigenvalue $\lambda_{\min }$, where $\mathbf{M}^{t} \mathbf{v}=\lambda_{\min } \mathbf{v}$. We efficiently update $\mathbf{v}$ iteratively using Locally Optimal Block Preconditioned Conjugate Gradient (LOBPCG) [17] with warm start as diagonal / off-diagonal terms are optimized. Experiments show that our computed graph metrics outperform metrics learned using competing methods in terms of classification tasks.

\section{REVIEW OF SPECTRAL GRAPH THEORY}

We consider an undirected graph $\mathcal{G}=\{\mathcal{V}, \mathcal{E}, \mathbf{W}\}$ composed of a node set $\mathcal{V}$ of cardinality $|\mathcal{V}|=N$, an edge set $\mathcal{E}$ connecting nodes, and a weighted adjacency matrix $\mathbf{W}$. Each edge $(i, j) \in \mathcal{E}$ has a positive weight $w_{i, j}>0$ which reflects the degree of similarity (correlation) between nodes $i$ and $j$. Specifically, it is common to compute edge weight $w_{i, j}$ as the exponential of the negative feature distance $\delta_{i, j}$ between nodes $i$ and $j[18]$ :

$$
w_{i, j}=\exp \left(-\delta_{i, j}\right)
$$

\footnotetext{
${ }^{1}$ As the inter-feature correlations tend to zero, only graph self-loops expressing relative importance among the $K$ features remain, and the generalized graph Laplacian matrix tends to diagonal.
} 
Using (1) means $w_{i, j} \in(0,1]$ for $\delta_{i, j} \in[0, \infty)$. We discuss feature distance $\delta_{i, j}$ in the next section.

There may be self-loops in graph $\mathcal{G}$, i.e., $\exists i$ where $w_{i, i}>0$, and the corresponding diagonal entries of $\mathbf{W}$ are positive. The combinatorial graph Laplacian [18] is defined as $\mathbf{L}:=\mathbf{D}-\mathbf{W}$, where $\mathbf{D}$ is the degree matrix - a diagonal matrix where $d_{i, i}=\sum_{j=1}^{N} w_{i, j}$. A generalized graph Laplacian [11] accounts for self-loops in $\mathcal{G}$ also and is defined as $\mathbf{L}_{g}=\mathbf{D}-\mathbf{W}+\operatorname{diag}(\mathbf{W})$, where $\operatorname{diag}(\mathbf{W})$ extracts the diagonal entries of $\mathbf{W}$. Alternatively we can write $\mathbf{L}_{g}=$ $\mathbf{D}_{g}-\mathbf{W}$, where the generalized degree matrix $\mathbf{D}_{g}=\mathbf{D}+\operatorname{diag}(\mathbf{W})$ is diagonal.

\section{GRAPH METRIC LEARNING}

\subsection{Graph Metric Matrices}

We first define the search space of metric matrices for our optimization framework. We assume that associated with each sample $i$ is a length- $K$ feature vector $\mathbf{f}_{i} \in \mathbb{R}^{K}$. A metric matrix $\mathbf{M} \in \mathbb{R}^{K \times K}$ defines the feature distance $\delta_{i, j}(\mathbf{M})$ — the Mahalanobis distance [1]between samples $i$ and $j$ as:

$$
\delta_{i, j}(\mathbf{M})=\left(\mathbf{f}_{i}-\mathbf{f}_{j}\right)^{\top} \mathbf{M}\left(\mathbf{f}_{i}-\mathbf{f}_{j}\right)
$$

We require $\mathrm{M}$ to be a positive definite (PD) matrix The special case where $\mathbf{M}$ is diagonal with strictly positive entries was studied in [10]. Instead, we study here a more general case: $\mathbf{M}$ must be a graph metric matrix, which we define formally as follows.

Definition 1. A PD symmetric matrix $\mathbf{M}$ is a graph metric if it is a generalized graph Laplacian matrix with positive edge weights and node degrees for an irreducible graph.

Remark: A generalized graph Laplacian matrix $\mathbf{M}$ with positive degrees means $m_{i, i}>0$; in graph terminology, each graph node $i$ may have a self-loop, but its loop weight $w_{i, i}$ must satisfy $w_{i, i}>$ $-\sum_{j \mid j \neq i} w_{i, j}$. Positive edge weights means $m_{i, j} \leq 0, i \neq j$. Irreducible graph [20] essentially means that any graph node can commute with any other node.

\subsection{Problem Formulation}

Denote by $\mathcal{S}$ the set of all graph metric matrices. We pose an optimization problem for $\mathbf{M}$ : find the optimal graph metric $\mathbf{M}$ in $\mathcal{S}$ leading to inter-sample distances $\delta_{i, j}(\mathbf{M})$ in 2- that yields the smallest value of a convex differential objective $Q\left(\left\{\delta_{i, j}(\mathbf{M})\right\}\right)$ :

$$
\min _{\mathbf{M} \in \mathcal{S}} Q\left(\left\{\delta_{i, j}(\mathbf{M})\right\}\right), \text { s.t. } \operatorname{tr}(\mathbf{M}) \leq C
$$

where $C>0$ is a chosen parameter. Constraint $\operatorname{tr}(\mathbf{M}) \leq C$ is needed to avoid pathological solutions with infinite feature distances, i.e., $\delta_{i, i}(\mathbf{M})=\infty$. For stability, we assume also that the objective is lower-bounded, i.e., $\min _{\mathbf{M} \in \mathcal{S}} Q\left(\left\{\delta_{i, j}(\mathbf{M})\right\}\right) \geq \kappa>-\infty$ for some constant $\kappa$.

Our strategy to solve (3) is to optimize M's diagonal and offdiagonal terms alternately using Frank-Wolfe iterations [16], where each iteration is solved as an LP until convergence. We discuss first the initialization of $\mathbf{M}$, then the two optimizations in order. For notation convenience, we will write the objective simply as $Q(\mathbf{M})$, with the understanding that metric $\mathbf{M}$ affects first the feature distances $\delta_{i, j}(\mathbf{M})$, which in turn determine the objective $Q\left(\left\{\delta_{i, j}(\mathbf{M})\right\}\right)$.

\footnotetext{
${ }^{2}$ By definition of a metric [19], $\left(\mathbf{f}_{i}-\mathbf{f}_{j}\right)^{\top} \mathbf{M}\left(\mathbf{f}_{i}-\mathbf{f}_{j}\right)>0$ if $\mathbf{f}_{i}-\mathbf{f}_{j} \neq \mathbf{0}$.
}

\subsection{Initialization of $M$}

We first initialize a valid graph metric $\mathbf{M}^{0}$ as follows:

1. Initialize each diagonal term $m_{i, i}^{0}:=C / K$.

2. Initialize off-diagonal terms $m_{i, j}^{0}, i \neq j$, as:

$$
m_{i, j}^{0}:= \begin{cases}-\epsilon & \text { if } j=i \pm 1 \\ 0 & \text { o.w. }\end{cases}
$$

where $\epsilon>0$ is a small parameter. Initialization of the diagonal terms ensures that constraints $\operatorname{tr}\left(\mathbf{M}^{0}\right) \leq C, \mathbf{M}^{0} \succ 0$ and $m_{i, i}^{0}>0$ are satisfied. Initialization of the off-diagonal terms ensures that $\mathbf{M}^{0}$ is symmetric and irreducible, and constraint $m_{i, j}^{0} \leq 0, i \neq j$, is satisfied; i.e., $\mathbf{M}^{0}$ is a generalized graph Laplacian matrix for graph with positive edge weights. We can hence conclude that initial $\mathbf{M}^{0}$ is a graph metric, i.e., $\mathbf{M}^{0} \in \mathcal{S}$.

\subsection{Optimization of Diagonal Terms}

When optimizing M's diagonal terms $m_{i, i}$, (3) becomes

$$
\begin{array}{ll}
\min _{\left\{m_{i, i}\right\}} & Q(\mathbf{M}) \\
\text { s.t. } & \mathbf{M} \succ 0 ; \quad \sum_{i} m_{i, i} \leq C ; \quad m_{i, i}>0, \forall i
\end{array}
$$

where $\operatorname{tr}(\mathbf{M})=\sum_{i} m_{i, i}$. Because the diagonal terms do not affect the irreducibility of matrix $\mathbf{M}$, the only requirements for $\mathbf{M}$ to be a graph metric are: i) $\mathrm{M}$ must be $\mathrm{PD}$, and ii) diagonals must be strictly positive.

\subsubsection{Gershgorin-based Reformulation}

To efficiently enforce the PD constraint $\mathbf{M} \succ 0$, we derive sufficient (but not necessary) linear constraints using the Gershgorin Circle Theorem (GCT) [14]. By GCT, each eigenvalue $\lambda$ of a real matrix $\mathbf{M}$ resides in at least one Gershgorin disc $\Psi_{i}$, corresponding to row $i$ of $\mathbf{M}$, with center $c_{i}=m_{i, i}$ and radius $r_{i}=\sum_{j \mid j \neq i}\left|m_{i, j}\right|$, i.e.,

$$
\exists i \text { s.t. } c_{i}-r_{i} \leq \lambda \leq c_{i}+r_{i}
$$

Thus a sufficient condition to ensure $\mathrm{M}$ is PD (smallest eigenvalue $\lambda_{\min }>0$ ) is to ensure that all discs' left-ends are strictly positive, i.e.,

$$
0<\min _{i} c_{i}-r_{i} \leq \lambda_{\min }
$$

This translates to a linear constraint for each row $i$ :

$$
m_{i, i} \geq \sum_{j \mid j \neq i}\left|m_{i, j}\right|+\rho, \quad \forall i \in\{1, \ldots, K\}
$$

where $\rho>0$ is a sufficiently small parameter.

However, GCT lower bound $\min _{i} c_{i}-r_{i}$ for $\lambda_{\min }$ is often loose. When optimizing M's diagonal terms, enforcing (8) directly means that we are searching for $\left\{m_{i, i}\right\}$ in a much smaller space than the original space $\{\mathbf{M} \mid \mathbf{M} \succ 0\}$ in (5), resulting in an inferior solution. As an illustration, consider the following example matrix $\mathbf{M}$ :

$$
\mathbf{M}=\left[\begin{array}{ccc}
2 & -2 & -1 \\
-2 & 5 & -2 \\
-1 & -2 & 4
\end{array}\right]
$$

Gershgorin disc left-ends $m_{i, i}-\sum_{j \mid j \neq i}\left|m_{i, j}\right|$ for this matrix are $\{-1,1,1\}$, of which -1 is the smallest. Thus the diagonal terms $\{2,5,4\}$ do not meet constraints 8 . However, $\mathbf{M}$ is PD, since its smallest eigenvalue is $\lambda_{\min }=0.1078>0$. 


\subsubsection{Gershgorin Disc Alignment}

To derive more appropriate linear constraints - thus more suitable search space when solving $\min _{\mathbf{M} \in \mathcal{S}} \mathbf{Q}(\mathbf{M})$, we examine instead the Gershgorin discs of a similar-transformed matrix $\mathbf{B}$ from $\mathbf{M}$, i.e.,

$$
\mathbf{B}=\mathbf{S M S}^{-1}
$$

where $\mathbf{S}=\operatorname{diag}\left(s_{1}, \ldots, s_{K}\right)$ is a diagonal matrix with scalars $s_{1}, \ldots, s_{K}$ along its diagonal, $s_{k}>0, \forall k . \quad \mathbf{B}$ has the same eigenvalues as $\mathbf{M}$, and thus the smallest Gershgorin disc left-end, $\min _{i} b_{i, i}-\sum_{j \mid j \neq i}\left|b_{i, j}\right|$, for $\mathbf{B}$ is also a lower bound for M's smallest eigenvalue $\lambda_{\min }$. Our goal is to derive tight $\lambda_{\min }$ lower bounds by adapting to good solutions to (5) - by appropriately choosing scalars $s_{1}, \ldots, s_{K}$ used to define similar-transformed $\mathbf{B}$ in 10 .

Specifically, given scalars $s_{1}, \ldots, s_{K}$, a disc $\Psi_{i}$ for $\mathbf{B}$ has center $m_{i, i}$ and radius $s_{i} \sum_{j \mid j \neq i}\left|m_{i, j}\right| / s_{j}$. Thus to ensure $\mathbf{B}$ is PD (and hence $\mathbf{M}$ is PD), we can write similar linear constraints as (8):

$$
m_{i, i} \geq s_{i} \sum_{j \mid j \neq i} \frac{\left|m_{i, j}\right|}{s_{j}}+\rho, \quad \forall i \in\{1, \ldots, K\}
$$

It turns out that given a graph metric $\mathbf{M}$, there exist scalars $s_{1}, \ldots, s_{K}$ such that all Gershgorin disc left-ends are aligned at the same value $\lambda_{\min }$. We state this formally as a theorem.

Theorem 1. Let $\mathbf{M}$ be a graph metric matrix. There exist strictly positive scalars $s_{1}, \ldots, s_{K}$ such that all Gershgorin disc left-ends of $\mathbf{B}=\mathbf{S M S}^{-1}$ are aligned exactly at the smallest eigenvalue, i.e., $b_{i, i}-\sum_{j \mid j \neq i}\left|b_{i, j}\right|=\lambda_{\min }, \forall i$.

In other words, for matrix $\mathbf{B}$ the Gershgorin lower bound $\min _{i} c_{i}-r_{i}$ is exactly $\lambda_{\min }$, and the bound is the tightest possible. The important corollary is the following:

Corollary 1. For any graph metric matrix $\mathbf{M}$, which by definition is $P D$, there exist scalars $s_{1}, \ldots, s_{K}$ where $\mathbf{M}$ is feasible using linear constraints in 11.

Proof. By Theorem 1, let $s_{1}, \ldots, s_{K}$ be scalars such that all Gershgorin disc left-ends of $\mathbf{B}=\mathbf{S M S}^{-1}$ align at $\lambda_{\min }$. Thus

$$
\forall i, \quad m_{i, i}-s_{i} \sum_{j \mid j \neq i} \frac{\left|m_{i, j}\right|}{s_{j}}=\lambda_{\min }>0
$$

where $\lambda_{\min }>0$ since $\mathbf{M}$ is PD. Hence $\mathbf{M}$ must also satisfy 11 for all $i$ for sufficiently small $\rho>0$.

Continuing our earlier example, using $s_{1}=0.7511, s_{2}=$ 0.4886 and $s_{3}=0.4440$, we see that $\mathbf{B}=\mathbf{S M S}^{-1}$ for $\mathbf{M}$ in 9) has all disc left-ends aligned at $\lambda_{\min }=0.1078$. Hence using these scalars and constraints 11, diagonal terms $\{2,5,4\}$ now constitute a feasible solution.

To prove Theorem 1, we first establish the following lemma.

Lemma 1. There exists a first eigenvector $\mathbf{v}$ with strictly positive entries for a graph metric matrix $\mathbf{M}$.

Proof. By definition, graph metric matrix $\mathbf{M}$ is a generalized graph Laplacian $\mathbf{L}_{g}=\mathbf{D}_{g}-\mathbf{W}$ with positive edge weights in $\mathbf{W}$ and positive degrees in $\mathbf{D}_{g}$. Let $\mathbf{v}$ be the first eigenvector of $\mathbf{M}$, i.e.,

$$
\begin{aligned}
\mathbf{M} \mathbf{v} & =\lambda_{\min } \mathbf{v} \\
\left(\mathbf{D}_{g}-\mathbf{W}\right) \mathbf{v} & =\left(\lambda_{\min } \mathbf{I}\right) \mathbf{v} \\
\mathbf{D}_{g} \mathbf{v} & =\left(\mathbf{W}+\lambda_{\min } \mathbf{I}\right) \mathbf{v} \\
\mathbf{v} & =\mathbf{D}_{g}^{-1}\left(\mathbf{W}+\lambda_{\min } \mathbf{I}\right) \mathbf{v}
\end{aligned}
$$

where $\lambda_{\min }>0$ since $\mathbf{M}$ is PD. Since the matrix on the right contains only non-negative entries and $\mathbf{W}$ is an irreducible matrix, $\mathbf{v}$ is a positive eigenvector by the Perron-Frobenius Theorem [21].

We now prove Theorem 1 as follows.

Proof. Denote by $\mathbf{v}$ a strictly positive eigenvector corresponding to graph metric matrix $\mathbf{M}$ 's smallest eigenvalue $\lambda_{\min }$. Define $\mathbf{S}=$ $\operatorname{diag}\left(1 / v_{1}, \ldots, 1 / v_{K}\right)$. Then,

$$
\mathbf{S M S}^{-1} \mathbf{S v}=\lambda_{\min } \mathbf{S v}
$$

where $\mathbf{S v}=\mathbf{1}=[1, \ldots, 1]^{\top}$. Let $\mathbf{B}=\mathbf{S M S}^{-1}$. Then,

$$
\mathbf{B} \mathbf{1}=\lambda_{\min } \mathbf{1}
$$

(14) means that

$$
b_{i, i}+\sum_{j \mid j \neq i} b_{i, j}=\lambda_{\min }, \quad \forall i
$$

Note that the off-diagonal terms $b_{i, j}=\left(v_{i} / v_{j}\right) m_{i, j} \leq 0$, since i) $\mathbf{v}$ is strictly positive and ii) off-diagonal terms of graph metric $\mathbf{M}$ satisfy $m_{i, j} \leq 0$. Thus,

$$
b_{i, i}-\sum_{j \mid j \neq i}\left|b_{i, j}\right|=\lambda_{\min }, \quad \forall i
$$

Thus defining $\mathbf{S}=\operatorname{diag}\left(1 / v_{1}, \ldots, 1 / v_{K}\right)$ means $\mathbf{B}=\mathbf{S M S}^{-1}$ has all its Gershgorin disc left-ends aligned at $\lambda_{\min }$.

Thus, using a positive first eigenvector $\mathbf{v}$ of a graph metric $\mathbf{M}$, one can compute corresponding scalars $s_{k}=1 / v_{k}$ to align all disc left-ends of $\mathbf{B}=\mathbf{S M S}^{-1}$ at $\lambda_{\min }$, and $\mathbf{M}$ satisfies (11) by Corollary 1 . Note that these scalars are signal-adaptive, i.e., $s_{k}$ 's depend on $\mathbf{v}$, which is computed from $\mathbf{M}$. Our strategy then is to derive scalars $s_{k}^{t}$,s from a good solution $\mathbf{M}^{t-1}$, optimize for a better solution $\mathbf{M}^{t}$ using scaled Gershgorin linear constraints 11 , derive new scalars again until convergence. Specifically,

1. Given scalars $s_{k}^{t}$ 's, identify a good solution $\mathbf{M}^{t}$ minimizing objective $Q(\mathbf{M})$ subject to 111 , i.e.,

$$
\begin{aligned}
& \min _{\left\{m_{i, i}\right\}} Q(\mathbf{M}) \\
& \text { s.t. } m_{i, i} \geq s_{i} \sum_{j \mid j \neq i} \frac{\left|m_{i, j}\right|}{s_{j}}+\rho, \forall i ; \quad \sum_{i} m_{i, i} \leq C
\end{aligned}
$$

2. Given $\mathbf{M}^{t}$, update scalars $s_{k}^{t+1}=1 / v_{k}^{t}$ where $\mathbf{v}^{t}$ is the first eigenvector of $\mathbf{M}^{t}$.

3 . Increment $t$ and repeat until convergence.

When the scalars in (16) are updated as $s_{k}^{t+1}=1 / v_{k}^{t}$ for iteration $t+1$, we show that previous solution $\mathbf{M}^{t}$ at iteration $t$ remains feasible at iteration $t+1$ :

Lemma 2. Solution $\mathbf{M}^{t}$ to 16 in iteration $t$ remains feasible in iteration $t+1$, when scalars $s_{i}^{t+1}$ for the linear constraints in 16 are updated as $s_{i}^{t+1}=1 / v_{i}^{t}, \forall i$, where $\mathbf{v}^{t}$ is the first eigenvector of $\mathbf{M}^{t}$.

Proof. Using the first eigenvector $\mathbf{v}^{t}$ of graph metric $\mathbf{M}^{t}$ at iteration $t$, by the proof of Theorem 1 we know that the Gershgorin disc leftends of $\mathbf{B}=\mathbf{S M}^{t} \mathbf{S}^{-1}$ are aligned at $\lambda_{\min }$. Since $\mathbf{M}^{t}$ is a feasible solution in 16, $\mathbf{M}^{t} \succ 0$ and $\lambda_{\min }>0$. Thus $\mathbf{M}^{t}$ is also a feasible solution when scalars are updated as $s_{i}=1 / v_{i}^{t}, \forall i$. 
The remaining issue is how to best compute first eigenvector $\mathbf{v}^{t}$ given solution $\mathbf{M}^{t}$ repeatedly. For this task, we employ Locally Optimal Block Preconditioned Conjugate Gradient (LOBPCG) [17], a fast iterative algorithm known to compute extreme eigenpairs efficiently. Further, using previously computed eigenvector $\mathbf{v}^{t-1}$ as an initial guess, LOBPCG benefits from warm start when computing $\mathbf{v}^{t}$, reducing its complexity in subsequent iterations [17].

\subsubsection{Frank-Wolfe Algorithm}

To solve [16, we employ the Frank-Wolfe algorithm [16] that iteratively linearizes the objective $Q(\mathbf{M})$ using its gradient $\nabla Q\left(\mathbf{M}^{t}\right)$ with respect to diagonal terms $\left\{m_{i, i}\right\}$, computed using previous solution $\mathbf{M}^{t}$, i.e.,

$$
\nabla Q\left(\mathbf{M}^{t}\right)=\left.\left[\begin{array}{c}
\frac{\partial Q(\mathbf{M})}{\partial m_{1,1}} \\
\vdots \\
\frac{\partial Q(\mathbf{M})}{\partial m_{K, K}}
\end{array}\right]\right|_{\mathbf{M}^{t}}
$$

Given gradient $\nabla Q\left(\mathbf{M}^{t}\right)$, optimization 16 becomes a linear program (LP) at each iteration $t$ :

$$
\begin{aligned}
& \min _{\left\{m_{i, i}\right\}} \operatorname{vec}\left(\left\{m_{i, i}\right\}\right)^{\top} \nabla Q\left(\mathbf{M}^{t}\right) \\
& \quad \text { s.t. } m_{i, i} \geq s_{i} \sum_{j \mid j \neq i} \frac{\left|m_{i, j}^{t}\right|}{s_{j}}+\rho, \quad \forall i ; \quad \sum_{i} m_{i, i} \leq C .
\end{aligned}
$$

where $\operatorname{vec}\left(\left\{m_{i, i}\right\}\right)=\left[\begin{array}{llll}m_{1,1} & m_{2,2} & \ldots & m_{K, K}\end{array}\right]^{\top}$ is a vector composed of diagonal terms $\left\{m_{i, i}\right\}$, and $m_{i, j}^{t}$ are off-diagonal terms of previous solution $\mathbf{M}^{t}$. LP (18) can be solved efficiently using known fast algorithms such as Simplex [15] and interior point method [22]. When a new solution $\left\{m_{i, i}^{t+1}\right\}$ is obtained, gradient $\nabla Q\left(\mathbf{M}^{t+1}\right)$ is updated, and LP 18 is solved again until convergence.

\subsection{Optimization of Off-diagonal Entries}

For off-diagonal entries of $\mathbf{M}$, we design a block coordinate descent algorithm, which optimizes one row / column at a time.

\subsubsection{Block Coordinate Iteration}

First, we divide $\mathbf{M}$ into four sub-matrices:

$$
\mathbf{M}=\left[\begin{array}{ll}
m_{1,1} & \mathbf{M}_{1,2} \\
\mathbf{M}_{2,1} & \mathbf{M}_{2,2}
\end{array}\right],
$$

where $m_{1,1} \in \mathbb{R}, \mathbf{M}_{1,2} \in \mathbb{R}^{1 \times(K-1)}, \mathbf{M}_{2,1} \in \mathbb{R}^{(K-1) \times 1}$ and $\mathbf{M}_{2,2} \in \mathbb{R}^{(K-1) \times(K-1)}$. Assuming $\mathbf{M}$ is symmetric, $\mathbf{M}_{1,2}=$ $\mathbf{M}_{2,1}^{\top}$. We optimize $\mathbf{M}_{2,1}$ in one iteration, i.e.,

$$
\min _{\mathbf{M}_{2,1}} Q(\mathbf{M}) \text {, s.t. } \mathbf{M} \in \mathcal{S}
$$

In the next iteration, a different row / column $i$ is selected, and with appropriate row / column permutation, we still optimize the first column off-diagonal terms $\mathbf{M}_{2,1}$ as in 201.

Note that the constraint $\operatorname{tr}(\mathbf{M}) \leq C$ in $(3)$ can be ignored, since it does not involve optimization variable $\mathbf{M}_{2,1}$. For $\mathbf{M}$ to remain in the set $\mathcal{S}$ of graph metric matrices, i) $\mathbf{M}$ must be PD, ii) $\mathbf{M}$ must be irreducible, and iii) $\mathbf{M}_{2,1} \leq \mathbf{0}$.

As done for the diagonal terms optimization, we replace the PD constraint with Gershgorin-based linear constraints. To ensure irreducibility (i.e., the graph remains connected), we ensure that at least one off-diagonal term (say index $\zeta$ ) in column 1 has magnitude at least $\epsilon>0$. The optimization thus becomes:

$$
\begin{array}{rl}
\min _{\mathbf{M}_{2,1}} & Q(\mathbf{M}) \\
\text { s.t. } & m_{i, i} \geq s_{i} \sum_{j \mid j \neq i} \frac{\left|m_{i, j}\right|}{s_{j}}+\rho, \forall i \\
& m_{\zeta, 1} \leq-\epsilon ; \quad \mathbf{M}_{2,1} \leq \mathbf{0}
\end{array}
$$

Essentially any selection of $\zeta$ in 21) can ensure $\mathbf{M}$ is irreducible. To encourage solution convergence, we select $\zeta$ as the index of the previously optimized $\mathbf{M}_{2,1}^{t}$ with the largest magnitude.

21) also has a convex differentiable objective with a set of linear constraints. We thus employ the Frank-Wolfe algorithm again to iteratively linearize the objective using gradient $\nabla Q\left(\mathbf{M}^{t}\right)$ with respect to off-diagonal $\mathbf{M}_{2,1}$, where the solution in each iteration is solved as an LP. We omit the details for brevity.

\section{EXPERIMENTS}

We evaluate our proposed metric learning method in classification performance. Specifically, the objective function $Q(\mathbf{M})$ we consider here is the graph Laplacian Regularizer (GLR) [18, 23]:

$$
\begin{aligned}
& Q(\mathbf{M})=\mathbf{z}^{\top} \mathbf{L}(\mathbf{M}) \mathbf{z}=\sum_{i=1}^{N} \sum_{j=1}^{N} w_{i, j}\left(z_{i}-z_{j}\right)^{2} \\
= & \sum_{i=1}^{N} \sum_{j=1}^{N} \exp \left\{-\left(\mathbf{f}_{i}-\mathbf{f}_{j}\right)^{\top} \mathbf{M}\left(\mathbf{f}_{i}-\mathbf{f}_{j}\right)\right\}\left(z_{i}-z_{j}\right)^{2} .
\end{aligned}
$$

A small GLR means that signal $\mathbf{z}$ at connected node pairs $\left(z_{i}, z_{j}\right)$ are similar for a large edge weight $w_{i, j}$, i.e., $z$ is smooth with respect to the variation operator $\mathbf{L}(\mathbf{M})$. GLR has been used in the GSP literature to solve a range of inverse problems, including image denoising [23], deblurring [24], dequantization amd contrast enhancement [25], and soft decoding of JPEG [26].

We evaluate our method with the following competing schemes: three metric learning methods that only learn the diagonals of $\mathbf{M}$, i.e., [27], [28], and [10], and two methods that learn the full matrix M, i.e., [6] and [29]. We perform classification tasks using one of the following two classifiers: 1) a k-nearest-neighbour classifier, and 2) a graph-based classifier with quadratic formulation $\min _{\mathbf{z}} \mathbf{z}^{\top} \mathbf{L}(\mathbf{M}) \mathbf{z}$ s.t. $z_{i}=\hat{z}_{i}, i \in \mathcal{F}, \mathcal{F} \subset\{1, \ldots, J\}$, where $\hat{z}_{i}$ in subset $\mathcal{F}$ are the observed labels. We evaluate all classifiers on wine ( 3 classes, 13 features and 178 samples), iris ( 3 classes, 4 features and 150 samples), seeds ( 3 classes, 7 features and 210 samples), and pb (2 classes, 10 features and 300 samples). All experiments were performed in Matlab R2017a on an i5-7500, 8GB of RAM, Windows 10 PC. We perform 2-fold cross validation 50 times using 50 random seeds (0 to 49) with one-against-all classification strategy. As shown in Table 1 our proposed metric learning method has the lowest classification error rates with a graph-based classifier.

Table 1. Classification error rates. (GB=Graph-based classifier.)

\begin{tabular}{|c|c|c|c|c|c|c|c|c|}
\hline \multirow{2}{*}{ methods } & \multicolumn{2}{|c|}{ iris } & \multicolumn{2}{c|}{ wine } & \multicolumn{2}{c|}{ seeds } & \multicolumn{2}{c|}{ pb } \\
\cline { 2 - 9 } & $\mathrm{kNN}$ & $\mathrm{GB}$ & $\mathrm{kNN}$ & $\mathrm{GB}$ & $\mathrm{kNN}$ & $\mathrm{GB}$ & $\mathrm{kNN}$ & $\mathrm{GB}$ \\
\hline$[27]$ & 4.61 & 4.41 & 3.84 & 4.88 & 7.30 & 7.20 & - & - \\
{$[28$} & 4.97 & 4.57 & 4.61 & 5.18 & 7.15 & 6.93 & 4.46 & 5.04 \\
{$[10]$} & 5.45 & 5.49 & 4.35 & 4.96 & 7.78 & 7.40 & 5.33 & 4.51 \\
\hline$[6]$ & 6.12 & 10.40 & $\mathbf{3 . 5 8}$ & 4.37 & $\mathbf{6 . 9 2}$ & 6.63 & 4.55 & 4.96 \\
{$[29]$} & $\mathbf{4 . 3 5}$ & 4.80 & 4.12 & 4.36 & 7.77 & 7.47 & $\mathbf{4 . 4 4}$ & 4.24 \\
Prop. & $\mathbf{4 . 3 5}$ & $\mathbf{4 . 1 2}$ & 4.27 & $\mathbf{4 . 1 9}$ & 7.10 & $\mathbf{6 . 6 1}$ & 4.8 & $\mathbf{4 . 2 3}$ \\
\hline
\end{tabular}




\section{REFERENCES}

[1] P. C. Mahalanobis, "On the generalized distance in statistics," Proceedings of the National Institute of Sciences of India, vol. 2, no. 1, pp. 49-55, April 1936.

[2] K. Q. Weinberger and L. K. Saul, "Distance metric learning for large margin nearest neighbor classification," Journal of Machine Learning Research, vol. 10, no. 2, pp. 207-244, Feb. 2009.

[3] G.-J. Qi, J. Tang, Z.-J. Zha, T.-S. Chua, and H.-J. Zhang, "An efficient sparse metric learning in high-dimensional space via $l_{1}$-penalized log-determinant regularization," in $I C M L$, June 2009, pp. 841-848.

[4] D. Lim, G. Lanckriet, and B. McFee, "Robust structural metric learning," in ICML, June 2013, pp. 615-623.

[5] W. Liu, C. Mu, R. Ji, S. Ma, J. R. Smith, and S.-F. Chang, "Low-rank similarity metric learning in high dimensions," in AAAI, Jan. 2015, p. 27922799.

[6] P. Zadeh, R. Hosseini, and S. Sra, "Geometric mean metric learning," in ICML, June 2016, pp. 2464-2471.

[7] N. Parikh and S. Boyd, "Proximal algorithms," Foundations and Trends in Optimization, vol. 1, no. 3, pp. 127-239, Jan. 2014.

[8] Y. Mu, "Fixed-rank supervised metric learning on Riemannian manifold," in AAAI, Feb. 2016, pp. 1941-1947.

[9] J. Zhang and L. Zhang, "Efficient stochastic optimization for low-rank distance metric learning," in $A A A I$, Feb. 2017, pp. 933-939.

[10] C. Yang, G. Cheung, and V. Stankovic, "Alternating binary classifier and graph learning from partial labels," in APSIPA, Nov. 2018, pp. 1137-1140.

[11] T. Biyikoglu, J. Leydold, and P. F. Stadler, "Nodal domain theorems and bipartite subgraphs," The Electronic Journal of Linear Algebra, vol. 13, pp. 344-351, Jan. 2005.

[12] Y. Bai, G. Cheung, F. Wang, X. Liu, and W. Gao, "Reconstruction-cognizant graph sampling using Gershgorin disc alignment," in ICASSP, May 2019, pp. 5396-5400.

[13] Y. Bai, F. Wang, G. Cheung, Y. Nakatsukasa, and W. Gao, "Fast graph sampling set selection using Gershgorin disc alignment," arXiv, 2019.

[14] R. S. Varga, Gershgorin and his circles. Springer, 2004.

[15] C. Papadimitriou and K. Steiglitz, Combinatorial Optimization. Dover Publications, Inc, 1998.

[16] M. Jaggi, "Revisiting Frank-Wolfe: Projection-free sparse convex optimization," in ICML, Jun. 2013, pp. 427-435.

[17] A. V. Knyazev, "Toward the optimal preconditioned eigensolver: Locally optimal block preconditioned conjugate gradient method," SIAM Journal on Scientific Computing, vol. 23, no. 2, pp. 517-541, 2001.

[18] D. I. Shuman, S. K. Narang, P. Frossard, A. Ortega, and P. Vandergheynst, "The emerging field of signal processing on graphs: Extending high-dimensional data analysis to networks and other irregular domains," IEEE Signal Processing Magazine, vol. 30, pp. 83-98, May 2013.

[19] M. Vetterli, J. Kovacevic, and V. Goyal, Foundations of Signal Processing. Cambridge University Press, 2014.
[20] M. Milgram, "Irreducible graphs," Journal Of Combinatorial Theory (B), vol. 12, pp. 6-31, Feb. 1972.

[21] R. Horn and C. Johnson, Matrix Analysis. Cambridge University Press, 2012.

[22] S. Boyd and L. Vandenberghe, Convex Optimization. Cambridge University Press, 2009.

[23] J. Pang and G. Cheung, "Graph Laplacian regularization for image denoising: Analysis in the continuous domain," IEEE Transactions on Image Processing, vol. 26, no. 4, pp. 17701785, April 2017.

[24] Y. Bai, G. Cheung, X. Liu, and W. Gao, "Graph-based blind image deblurring from a single photograph," IEEE Transactions on Image Processing, vol. 28, no. 3, pp. 1404-1418, March 2019.

[25] X. Liu, G. Cheung, X. Ji, D. Zhao, and W. Gao, "Graphbased joint dequantization and contrast enhancement of poorly lit JPEG images," IEEE Transactions on Image Processing, vol. 28, no. 3, pp. 1205-1219, March 2019.

[26] X. Liu, G. Cheung, X. Wu, and D. Zhao, "Random walk graph Laplacian-based smoothness prior for soft decoding of JPEG images," IEEE Transactions on Image Processing, vol. 26, no. 2, pp. 509-524, Feb. 2017.

[27] X. Zhu, Z. Ghahramani, and J. Lafferty, "Semi-supervised learning using Gaussian fields and harmonic functions," in ICML, Aug. 2003, pp. 912-919.

[28] Y. Mao, G. Cheung, C.-W. Lin, and Y. Ji, "Joint learning of similarity graph and image classifier from partial labels," in APSIPA, Dec. 2016, pp. 1-4.

[29] W. Hu, X. Gao, G. Cheung, and Z. Guo, "Feature graph learning for 3d point cloud denoising," arXiv, 2019. 